\title{
Molecular Epidemiology of Cancer
}

National Cancer Institute

\section{Source}

National Cancer Institute. Molecular Epidemiology of Cancer. NCI Thesaurus. Code C19965.

Epidemiologic research on the environmental and genetic determinants of cancer.

(Bypass Budget) 